\title{
Nous, les maîtres d'école de la montagne... récits de vocation pédagogique
}

\section{Patrick Cabanel}

\section{(2) OpenEdition}

1 Journals

\section{Édition électronique}

URL : http://journals.openedition.org/trema/1866

DOI : 10.4000/trema.1866

ISSN : 2107-0997

\section{Éditeur}

Faculté d'Éducation de l'université de Montpellier

\section{Édition imprimée}

Date de publication : 1 décembre 1997

Pagination : 69-83

ISSN : 1167-315X

\section{Référence électronique}

Patrick Cabanel, « Nous, les maîtres d'école de la montagne... récits de vocation pédagogique », Tréma [En ligne], 12-13 | 1997, mis en ligne le 01 décembre 1997, consulté le 19 avril 2019. URL : http:// journals.openedition.org/trema/1866 ; DOI : 10.4000/trema.1866

Ce document a été généré automatiquement le 19 avril 2019.

Trema 


\title{
Nous, les maîtres d'école de la montagne... récits de vocation pédagogique
}

\author{
Patrick Cabanel
}

à Rosa PELLET (1893-1917), institutrice de hameau (Malzieu-Forain, Prunières, col de Montmirat), en Lozère, morte de la tuberculose.

1 «Pourquoi donc les départements les plus pauvres et les plus en retard sous le rapport de l'instruction, c'est-à-dire ceux qui à ce double titre, ne devraient fournir que peu de fonctionnaires de cette nature [...] donnent-ils une telle affluence de candidats? Au contraire, dans les parties de la France les mieux favorisées au point de vue intellectuel, et sous le rapport de la fortune [...], vous avez infailliblement beaucoup moins de sujets se destinant à la carrière de l'enseignement primaire. [...] Vous pouvez aller dans n'importe quelle partie de la France, partout vous trouverez des pédagogues originaires de nos endroits. Soit au nord, soit au midi, si vous êtes Lozérien, vous pouvez être sûr de trouver un compatriote ou à l'église ou à l'école. Chez nous, pas un village, pas un hameau qui n'ait donné le jour à un ecclésiastique ou à un instituteur ». Ces remarques sont émises en 1886 par un habitant du Malzieu (Lozère), dans les colonnes de l'hebdomadaire L'Auvergnat de Paris. Près de vingt ans plus tard, le nouveau sénateur catholique du département, Emmanuel de Las Cases, confirme : «La Lozère, qui est une pépinière de prêtres et de religieux, est aussi une pépinière de fonctionnaires. Pas une famille qui n'en compte quelquesuns. La même maison fournit à la fois des jésuites à la religion et des instituteurs au gouvernement ${ }^{1}$ ».

2 La terre des prêtres est donc aussi celle des instituteurs, en Lozère comme dans tout le sud du Massif Central, montagne à pédagogues comme l'étaient les Alpes et leurs régents aux trois plumes. Bien des documents permettraient de retracer les destins des maîtres de l'école rurale, hommes et femmes, religieux et laïques, à commencer par les dossiers personnels conservés aux Archives Départementales (et librement consultables, pour les 
plus anciens). Il n'a manqué à leurs cohortes ni des aventuriers (Pierre Bergogne, embarqué en 1902 pour Saint-Pierre-et-Miquelon, dans les rangs de la Mission Laïque, ou ces dizaines de Frères des Écoles Chrétiennes passés à la même époque en Amérique du Sud), ni cette pure figure de « martyr » laïque, Marthe Dupeyron, morte à vingt-et-un ans, avec sa jeune sœur, dans une tourmente de neige sur le flanc du Mont Lozère, en janvier 1941, alors qu'elle tentait de regagner son poste, contre toutes les objurgations ${ }^{2}$

On trouve aussi parmi les maîtres lozériens des mémorialistes soucieux de conserver le souvenir d'une saison ou d'une vie consacrées à l'encre et à la craie. Huguette Bastide a publié en 1969 Institutrice de village ${ }^{3}$, un portrait au vitriol de la vie dans diverses écoles rurales de la Lozère au début des années 1960 : la pauvreté, pour ne pas dire une certaine misère, économique, psychologique, culturelle, semble régner partout, dans ce couple fragile de tout jeunes maitres originaires de Mende et se sentant en exil dans les hameaux de la Margeride, comme dans les familles de leurs élèves, qui semblent radicalement indifférentes à leur destin scolaire. «Boue, cailloux, gadoue» (p. 128) : c'est sur ce mode que la jeune femme, écrasée par les conditions rudimentaires de la vie dans l'hiver lozérien, décline une expérience traumatisante. Le même hiver, la même dureté des conditions de vie en Margeride ont inspiré un livre au son tout différent, Éveilleur d'âmes. La première classe d'un instituteur de campagne $e^{4}$. L'auteur, Marcel Boyer, est né en 1921 et a grandi dans la commune de Rimeize ; il a volontairement mêlé ses souvenirs d'écolier et ceux de son premier poste d'instituteur (à Arcomie). Il en résulte, me semble-t-il, un très grand témoignage sur l'école rurale: les notations les plus concrètes s'y insèrent dans un véritable roman d'initiation, qui conduit un jeune homme de Marvejols à s'enraciner dans un hameau de la Margeride neigeuse jusqu'à souhaiter ne plus le quitter au terme de l'année scolaire. Le maître d'école s'est arrêté à Eboli pourrait être le sous-titre de cette fiction autobiographique. La langue de Marcel Boyer, de surcroît, par un phénomène de mimétisme sans doute involontaire, calque de près ce "parler primaire ", très surveillé, un peu naïf, un rien trop sérieux, que Charles Péguy avait décrit précisément à partir de Jean Coste (1902), le roman autobiographique de l'Aveyronnais Antonin Lavergne. Si, du reste, on élargit la collecte des souvenirs d'instituteurs à l'ensemble du Massif Central, on peut citer aux côtés du même Jean Coste l'émouvant et superbe Un pâtre du Cantal, de Pierre Besson (1914), Jean Francesou. Roman d'un petit berger (1897), du Lozérien devenu instituteur à Paris Jean-François Roux et Henri Gouttebel instituteur (1971), roman autobiographique de l'universitaire auvergnat Lucien Gachon. Citons encore, plus près de nous, Le pays des asphodèles, d'Adrienne Durand-Tullou (1989), autre récit d'un premier poste, ici dans les Causses gardois, et Les Hussards Verts, un ensemble de témoignages sur les normaliens ayant débuté après 1945 dans les monts de Lacaune (Tarn) ${ }^{5}$. L'extension de l'aire de collecte aux Alpes permettrait d'ajouter à la liste le célèbre Une soupe aux herbes sauvages, d'Émilie Carles (1977) et Un parfum d'encre et de craie, de la queyrassienne Céleste Fournier (1996). Du côté des instituteurs libres, on retiendra l'émouvant Monsieur Brajon, maître d'école, qui renvoie une fois encore à la misère des conditions de vie dans la Lozère rurale de l'entre-deux-guerres, ici aux portes de Mende ${ }^{6}$.

\section{Conditions et institutions du recrutement pédagogique}

4 Il y aurait beaucoup à dire à partir de ce corpus, que les historiens de la ruralité sont en droit de convoquer aussi bien que ceux de la scolarisation. Mais cela dépasserait de beaucoup les dimensions d'un bref article, sauf à résumer, sous la forme décevante d'une 
bibliographie commentée, ces témoignages de qualité dont je viens de donner une liste non exhaustive. Aussi ai-je choisi de ne retenir qu'un moment de ces destinées, mais non le moins important, certes : celui de la vocation. C'est à dessein que j'emploie un terme qui appartient au vocabulaire religieux, mais aussi à la manière populaire, toujours vivante, de désigner les motivations qui conduisent à embrasser une carrière enseignante. On vient de voir que la vocation religieuse et la vocation pédagogique semblaient bien voisines dans la Lozère du $\mathrm{XIX}^{\mathrm{e}}$ siècle ${ }^{7}$, On peut même parler de systèmes jumeaux, et rivaux. À commencer par les mécanismes de succession, restés radicalement inégalitaires en dépit de l'institution du Code Civil : la propriété revenant à un héritier unique (fût-ce un fils cadet ou un gendre), les autres enfants sont désintéressés de l'héritage par ce que l'on appelait sous l'Ancien Régime la "légitime», et contraints d'aller chercher ailleurs les moyens de vivre. L'Église catholique offrait de longue date un destin enviable à ces exclus de la terre et du mariage ; au XIX ${ }^{e}$ siècle, elle multiplie les possibilités, jusqu'aux plus modestes, au sein de ses innombrables congrégations. Les Frères des Écoles Chrétiennes, les Petits Frères de Marie, les Frères du Sacré-Coeur recrutent par milliers au sud du Massif central, les vocations d'instituteurs religieux. Des dizaines de congrégations féminines, telles la Présentation de Marie ou les sœurs de Saint-Joseph des Vans, fondées par l'Ardéchoise Marie Rivier et la Lozérienne MarieThérèse Castanier, en font de même pour les femmes.

5 Une vive concurrence se fait jour, toutefois, avec l'institution du Brevet primaire, puis des Écoles normales : l'État, à son tour, offre une porte de sortie honorable aux cadets des familles rurales. La population protestante des Cévennes va s'y engouffrer, mais aussi les familles nombreuses du Gévaudan catholique. Il est peu probable, contrairement à l'affirmation du sénateur de Las Cases, qu'une même famille donne, au pire moment de l'affrontement entre les deux écoles, à la fois des Frères des Écoles Chrétiennes et des instituteurs laïques. Il y aurait là un véritable trait de schizophrénie familiale, alors que la réalité a dû être à la spécialisation des maisons, les terres blanches ayant compté leurs «bleus ». Et, on le sait bien, une fois un frère aîné, ou un oncle, entré dans l'une des institutions, il n'est pas rare qu'un membre plus jeune de la famille suive ses traces, cédant à diverses pressions, dont la moins efficace n'est pas le mimétisme, bénéficiant de livres, de pistes, d'appuis. Très vite se constituent ainsi des segments, voire de véritables épanouissements, dynastiques : l'administration comme les congrégations religieuses ne se montrent du reste nullement insensibles, dans l'appréciation d'un dossier, à la présence dans leurs cadres de parents du candidat, garants d'une disposition et d'une probable fidélité.

6 L'Église et l'École publique ont été, au cours du XIX siècle, de plus en plus explicitement rivales dans leur recrutement. La laïcisation du personnel dans les écoles publiques (loi Goblet de 1886), la multiplication des écoles rurales, jusque dans les hameaux, rendent nécessaire un recrutement relativement massif d'enseignants des deux sexes, alors que les diocèses et les instituts religieux ont déjà établi de solides réseaux, qu'ils vont renforcer en recourant notamment à la gratuité totale des études primaires et secondaires pour ceux et celles qui s'engagent à leur consacrer leur vie. Les champions des deux systèmes, prêtres, vicaires, frères et sœurs d'une part, instituteurs laïques de l'autre, cherchent à repérer, à séduire, à former, les mêmes cibles: les enfants les plus doués d'une génération. Les aptitudes sont connues de tous : à l'école publique comme au catéchisme, chacun a pu établir son classement, dont les noms se recoupent évidemment ${ }^{8}$ , et rêver de faire une recrue pour le séminaire ou l'école normale. Les choses se corsent 
du fait que, de 1850 à 1906, une disposition très suivie de la loi Falloux (l'article 66) permet à tout ecclésiastique de réunir librement jusqu'à quatre élèves auxquels il enseigne les rudiments du latin - en réalité, une véritable préparation à l'enseignement secondaire. Les instituteurs ne manquent pas de dénoncer aux inspecteurs primaires et d'Académie cette concurrence à leurs yeux déloyale, que leur font de jeunes vicaires, frais émoulus du grand séminaire et avides d'activité intellectuelle. Ainsi, en 1892, à Allenc (Lozère), où le vicaire aurait enseigné, à raison de quatre heures quotidiennes, le français à quatre enfants. Ce sont là, écrit l'instituteur, " quatre ou cinq de mes élèves de l'âge scolaire et des plus intelligents, de préférence ceux que je prépare à divers examens. [...] Pradel Guillaume, futur candidat à l'École normale, me remettait le trente novembre les livres que M. le Maire et moimême lui avions procurés, en me disant qu'il ne venait plus en classe, que son père avait besoin de lui. Hier matin, le jeune Galtier, candidat au prochain examen du Certificat d'études venait chercher ses effets classiques, devant également travailler avec son père, mais en réalité pour aller, ainsi que Pradel, en classe chez le vicaire ${ }^{9} \%$.

Diverses biographies de religieux rapportent de telles rivalités, tranchées au profit de l'Église: Charles Rocher (Cocurès, 1883), renonce à la bourse que lui promet son instituteur pour entrer dans la congrégation des Assomptionnistes; une jeune fille du Causse Méjan, promise à l'École normale par son institutrice, s'ingénie à échouer au certificat d'études pour échapper à la «laïque »: trouvant dans sa maison une vie de sainte Thérèse d'Avila, elle songe à partir pour Avila quand elle apprend l'existence du carmel de Mende, où elle entre bientôt ${ }^{10}$ !

L'école publique, plus tard venue dans le recrutement, aurait-elle cherché à imiter les institutions cléricales? Le parallèle a souvent été ébauché, et mérite d'être un peu systématiquement repris autour des vocations et de leur recrutement. Comment comprendre vraiment l'exceptionnel succès du certificat d'études, sans le rapprocher de la Première communion dont il semble l'équivalent laïque (outre que la communion est elle-même un examen couronnant les années de catéchisme) : passé en mai (le mois de Marie!) ou juin, comme son aînée, il est un rite de passage au sortir de l'enfance, accompli sensiblement au même âge que son antique rivale, et il finit par donner lieu à des rituels comparables (dons du parrain ou de la marraine, repas de famille, voyages). Il est l'occasion pour l'inspecteur primaire, ou d'Académie, d'entamer une tournée des écoles, comme le fait l'évêque pour les églises dans ses tournées de confirmation. Le haut personnage, alors, n'hésite pas à jouer au faiseur de destins en repérant publiquement des enfants et en leur intimant, ou à leurs familles, de poursuivre des études jusqu'aux Écoles normales. Le cardinal Bourret, évêque de Rodez à la fin du XIX siècle, s'était rendu célèbre par ces intimations ${ }^{11}$. Mais voici l'inspecteur d'Académie de la Lozère en visite à l'école de Sainte-Colombe-de-Peyre, au printemps 1892. Son attention est attirée par le jeune Jean-Augustin Dalle :

"Il demanda à l'élève d'aller chercher son père. Il fit part à ce dernier de l'impression qu'il avait ressentie devant l'intelligence et le savoir de son fils: Jean-Augustin perdait son temps à Sainte-Colombe; un élève aussi doué devait absolument poursuivre ses études et d'abord au Cours Complémentaire de Marvejols. Là où les instituteurs avaient échoué, l'inspecteur réussit: la famille, convaincue, se mit à l'ouvrage pour préparer le trousseau du futur étudiant ».

Jean-Augustin Dalle (1876-1944), entré deuxième à l'École normale de Mende, directeur de l'école de garçons d'Aumont-Aubrac, s'est fait l'historien de sa région natale à travers diverses publications : le groupe scolaire d'Aumont porte désormais son nom ${ }^{12}$. 
10 On sait combien de "vocations », si bien nommées, sont nées d'un tel geste d'impérative confiance publiquement délivré par le représentant suprême de l'autorité pédagogique dans les départements : Lucien Gachon, Lucie Mazauric, ÉmilieCarles, Jean Cornec, entre tant d'autres, se sont fait l'écho de ce qui fut le point de départ de bien des sagas de boursiers ${ }^{13}$. Derrière l'inspecteur, l'instituteur lui-même est invité à recruter.

"Vous savez, messieurs, qu'on se plaint partout du recrutement de plus en plus difficile des écoles normales. Chaque année, le nombre des candidats diminue. Aussi, M. l'inspecteur d'académie, qui déplore cet état de choses et qui craint qu'il ne nuise dans un avenir prochain au bon renom et aux intérêts du corps enseignant, m'a chargé de faire un pressant appel à votre incontestable dévouement. Vous pouvez, dans vos écoles et autour de vous, rechercher des vocations, les susciter même, et, en tout cas, faire une active propagande ",

11 s'exclame un inspecteur primaire dans une conférence pédagogique mise en scène par Antonin Lavergne ${ }^{14}$. "Ne voit-on pas les curés et les vicaires recruter des élèves dans leurs paroisses pour les établissements ecclésiastiques? Pourquoi l'instituteur n'en recruterait-il pas pour l'Université, pour l'État", estime dans un rapport de 1892 le député Charles Dupuy, futur ministre de l'Instruction publique, que sa naissance au Puy-en-Velay avait dû familiariser avec l'intensité du recrutement ecclésiastique ${ }^{15}$.

12 Les cours complémentaires, dont le développement est trop lent dans les zones "cléricales" (les municipalités doivent en effet solliciter la création de ces cours, et prendre à leur charge une partie des frais, ce qui explique une géographie très différenciée en fonction des positions idéologiques), sont compris comme un moyen pour la République et son école de se recruter : en 1920, des Comités de patronage (dont on ignore malheureusement tout) sont même mis en place auprès des cours complémentaires de la Lozère pour aider à leur développement. À l'inverse, il semble qu'assez fréquemment des maitres de qualité, placés à la tête d'écoles de chefs-lieux de commune, aient outrepassé leur mission en préparant leurs meilleurs élèves dans l'optique, même lointaine, de l'École normale: l'inspecteur d'Académie de Mende reconnaît en 1900 qu'il a dû "décourager certaines institutrices et certains instituteurs publics qui avaient pris l'habitude de substituer, en vue de la préparation aux examens et aux concours, dans les divisions supérieures de leur école, l'enseignement primaire supérieur à l'enseignement primaire proprement dit qui leur est demandé». Tel fut le cas de Justine Bastide (1869), fille de cordonnier, directrice de l'école de filles du Malzieu avant de prendre la tête du Cours complémentaire de filles de Vialas, qui rêvait de multiplier les institutrices parmi ses élèves, et parvint à faire décrocher trois brevets supérieurs, entre 1900 et 1910, à partir de sa seule école primaire du Malzieu, transformée en centre de préparation officieux à ce prestigieux examen ${ }^{16}$ !

\section{Prendre un état : le récit de vocation pédagogique}

13 Telles sont les conditions du recrutement des futurs instituteurs. Quels furent, chez les enfants concernés, les nécessités ou les désirs qui ont pu les mener à répondre positivement aux efforts entrepris par les maitres et la République ? Catégorie aisément identifiable, mais très particulière, les enfants d'instituteurs représentent un peu moins du cinquième des effectifs dans les Écoles Primaires Supérieures de filles et de garçons de Mende, voies royales pour les Écoles normales : 17 \% pour les garçons, près de $19 \%$ pour les filles (et $18 \%$ des 514 candidats aux bourses d'enseignement primaire supérieur entre 1912 et 1929) ${ }^{17}$. On sait que le taux d'endorecrutement parmi les officiers de l'armée ou les 
pasteurs protestants tourne également, à l'époque, autour de $20 \%$ : ce qui montre bien que les « hussards noirs ", sans constituer une caste, appartiennent à ce type de métiers respectés, mais clairement séparés du reste de la société, qui s'autoreproduisent en partie. On peut penser aussi que les enfants d'instituteurs sont invités, même inconsciemment, à répéter la vocation paternelle ou maternelle, en achevant ainsi de la légitimer : je renvoie à ce propos à de belles analyses proposées par Mona Ozouf ou Maurice Agulhon, enfants d'instituteurs ${ }^{18}$.

Quant aux autres, la majorité, il est bien difficile de déterminer les cheminements de leur vocation, sauf à reprendre la méthode magistralement mise en œuvre par Jacques et Mona Ozouf : l'envoi de questionnaires systématiques ${ }^{19}$, qu'il était vain de tenter pour ce modeste article. Au risque d'encourir le reproche classique d'impressionnisme, je me suis donc résolu à utiliser les témoignages écrits qui nous ont été conservés. Les deux plus anciens, du Lozérien Jean-François Roux, né en 1852, et du Cantalien Pierre Besson, né en 1873, sont précisément des récits de vocation : leur texte ne va pas au-delà des années de formation. Dans les deux cas, il s'agit d'enfances misérables passées dans les hameaux de la montagne; les familles sont nombreuses et pauvres, les enfants tôt "placés » ou «loués », quelquefois assez loin de leur domicile : ils gardent les troupeaux en échange de la nourriture et d'une très modeste somme d'argent. Les maîtres défilent, souvent trop sévères (mais pas plus que le père : il y a du Padre Padrone dans ces souvenirs) ${ }^{20}$; la neige envahit le récit, avec les derniers loups. Cet univers étouffant ne laisse apparaître que deux portes de sortie, qui fascinent les enfants : l'émigration à Paris (nous sommes au pays des bougnats, ces Auvergnats du sud devenus porteurs de charbon et garçons de café dans la capitale), et l'instruction. Pierre Besson rêve de suivre ses oncles et tous les Cantaliens qui ont fait fortune à Paris ; Jean-François Roux est loué dans les années 1860 chez des "Parisiens", des Lozériens enrichis dans le commerce et qui ont acheté des propriétés dans leur pays natal pour y redevenir paysans. En septembre 1872, parce qu'il ne supporte plus l'autoritarisme de son père, scieur de long, qui l'avait rappelé auprès de lui ${ }^{21}$, il suit un de ces «Parisiens » dans la capitale. Il lui faut quatre années de déboires, de chômage, d'emplois précaires, pour parvenir à entrer dans le monde scolaire. Arrivé analphabète à Paris, il apprend à lire et écrire auprès d'un compagnon de travail (le comptable d'un magasin de chaussures, rue de Rennes, ancien instituteur) puis grâce à des cours du soir. Il doit émigrer dans l'Aisne pour être accepté comme adjoint d'enseignement, chez divers maitres titulaires qui lui imposent de pendre pension à leur domicile, ce qui réduit à presque rien les économies qu'il espérait réaliser. Sa troisième candidature au brevet de capacité, en juillet 1880, débouche sur une brillante réussite, qui lui ouvre les portes des écoles communales de la ville de Paris. Il peut alors, huit ans après, venir retrouver les siens, pour leur faire part de son projet de mariage avec une Parisienne. Devenu instituteur parisien, et presque un notable, Jean-François Roux n'oublie pas son département: il participe dès ses débuts à l'Association lozérienne, la première société d'originaires de la Lozère, fondée à Paris en 1880 par le député républicain Théophile Roussel, et en devient premier vice-président dès 1883 et président d'honneur en 1886.

La passion de l'instruction n'a cessé de l'habiter, qu'il dispute ses brebis aux loups de la Margeride ou qu'il batte le pavé parisien. Sa première classe est tenue par une "béate ", presque une religieuse. Il la fréquente l'hiver, lorsque les troupeaux sont à l'étable. Il n'y apprend que le catéchisme et l'Évangile, la lecture et l'écriture étant un luxe réservé, à ses dires, aux enfants des grands propriétaires. À treize ans, placé à l'année chez un 
propriétaire de Rimeize, il a le loisir d'aller au cours du soir dirigé par l'instituteur des Estrets, à quatre kilomètres. Il apprend à lire, mais doit bientôt renoncer, pour ne pas avoir à traverser les domaines d'une riche propriétaire qui vient de mourir : «Il lui sembla voir, dans son sommeil, se promener dans ses propriétés la défunte richissime ». À partir de l'émigration à Paris, sa vie n'est plus qu'une suite de postes et de préparations aux examens. Et, d'une certaine façon, il n'a plus grand chose à en dire: la carrière des instituteurs heureux n'a pas d'histoire (Jean Coste est malheureux, on le sait). S'il en vient à publier des mémoires, en 1897 (le livre, illustré, est réédité en 1901, ce qui annonce un certain succès), c'est pour faire œuvre de moraliste, dans la pure tradition de l'école républicaine. C'est du reste un inspecteur primaire de Paris qui préface l'ouvrage, en invitant le lecteur à tirer profit de l'histoire ( La meilleure leçon de morale, celle qui résulte de l'exemple). Notre but ", écrit Roux : "nous avons voulu, en racontant une histoire absolument authentique, montrer qu'avec du travail et une bonne conduite on peut arriver sinon à la fortune, du moins à occuper une place honorable et utile dans la société ». Ses derniers mots interpellent encore : «Lecteurs, qui que vous soyez, de haute origine ou non, ayez du caractère, fixez-vous un but honorable, pour y voler tout droit et vous assurer l'avenir - comme le fit le pauvre petit berger dont notre livre vous raconte l'histoire». Trait caractéristique: l'autobiographie de l'instituteur se présente comme un conte pédagogique, une leçon de morale seulement un peu délayée, le temps de raconter un destin exemplaire. Ainsi fait Marcel Boyer, un siècle après Roux, dans Éveilleur d'âmes ${ }^{22}$. On est toujours dans l'univers scolaire; le Roman d'un petit berger pourrait être (a peut-être été, à Paris) un livre de distribution des prix.

Ainsi, et mieux encore, du texte de Pierre Besson. Ce normalien d'Aurillac, devenu professeur d'École primaire supérieure (à Murat) puis secrétaire d'inspection académique, a été invité à rédiger ses souvenirs par un inspecteur d'académie honoraire auquel il faisait visiter sa commune natale de Cheylade, dont il était, en début de carrière, l'instituteur. Les deux premiers chapitres, «Un caméristat dans le Cantal » et «Prendre un état », sont publiés en 1903 et 1904 dans ce temple de la pédagogie républicaine qu'est La Revue Pédagogique $e^{23}$. La rédaction accompagne le premier texte d'une note dans laquelle elle se défend d'avoir publié un texte qui serait mieux à sa place dans une revue de Folk-lore (sic) : pourquoi s'interdire "d'accueillir, de temps à autre, quelque récit, quelque notation d'impressions personnelles ayant d'ailleurs une valeur documentaire, comme c'est ici le cas. [...] En lisant les pages suivantes on ne regrettera qu'une chose, c'est de ne point posséder, pour chaque canton de France, une peinture aussi expressive, en son poétique réalisme, de nos écoliers paysans ». Pierre Besson ne tire un livre de ces articles que dix années plus tard. Son titre, Un pâtre du Cantal, comme sa composition (près des deux-tiers du livre décrivent une saison passée comme bouvier dans les burons de la montagne), sont curieusement trompeurs : on croit se trouver en présence d'une autobiographie rurale, annonçant les classiques nostalgiques des années 1970, alors qu'il s'agit bien du récit d'une vocation enseignante radicale, presque tragique, contre la misère et la dépendance. Pourquoi, dès lors, cet excursus,pour ne pas dire ce hors-sujet ruralisant? Parce qu'il y a déjà une demande en ce sens dans le public ? Parce que Pierre Besson tient à revenir une dernière fois, par le papier, à ce monde qui fut celui des siens, et qu'il a abandonné, avec la culpabilité sourde des "traitres" que furent peu ou prou les boursiers (Cf. Le Cheval d'orgueil, de Pierre-Jakez Hélias)? Ou encore parce qu'il retrouve un vieux schéma, chrétien ou laïque, du roman d'initiation, le passage par la tentation que le héros devra dominer pour s'affirmer? «Depuis, pas un printemps n'a passé sans venir me tourmenter et me dire: 〈Viens, laisse là cahiers et livres, les prés fleurissent et les bois s'éveillent ! 〉». Et comme la chèvre de Monsieur Seguin, le petit Pierre a décidé un jour de s'en aller dans la 
liberté des montagnes, en laissant là ses livres. "Au fait, dit ma grand-mère, si nous lui laissions manger un peu de vache enragée ». C'est sur ces mots que se refermait le second article de La Revue Pédagogique. Une saison de «loue» a suffi à ramener à l'école le buissonnier : or c'est elle que raconte longuement le livre, buissonnier à son tour !

Un pâtre du Cantal nous intéresse ici pour ce qu'il dit d'une vocation d'instituteur. Et qu'il dit avec une force particulière : on entre dans l'enseignement pour échapper à un très vieux malheur, et parce que l'on est comme ensorcelé par l'univers qui s'y dévoile ${ }^{24}$. La "vocation " à l'enfant est assurée par sa propre mère, mourante, qui le presse de «prendre un état »:

"Tu pleures, petit Pierre; il faut bien pourtant que je te dise avant de mourir ce que tu dois savoir! Oui, tu gagneras ton pain chez les autres. Tu mangeras la soupe noire de la ferme, le petit lait clair et fade comme l'eau, le croûton sec qu'on te mettra dans le sac [...]. Le soleil te brûlera la figure, te noircira le cou: Reste dehors! te dira ton maitre. Il pleut, il tonne: Reste dehors! Il neige: Reste dehors! toujours dehors, à la rage du temps! [...] Je mourrais contente, vois-tu, si je savais qu'un jour tu occuperas une place de quelque chose, maître d'école, par exemple. Tu serais toujours à l'abri, propre et sec, on t'appellerait Monsieur, et tu aurais une retraite à la fin. [...] Si tu pouvais prendre un état, pauvre Pierre, et n'avoir pas à suivre les maîtres, toujours dehors, à la rage du temps! [...] Ah! si tu savais! si tu savais! Comme tu travaillerais! Promets-moi que tu apprendras bien tes livres, que tu deviendras savant pour échapper au sort maudit des travailleurs de terre."

"Oui, mère, dis-je en sanglotant, je travaillerai, je t'assure ".

La mère meurt, et l'enfant, écrasé de chagrin, peine à se remettre à ses études : « Une nuit je crois entendre ma mère me renouveler ses conseils, et me redire avec plus d'insistance: ‘ Si tu pouvais prendre un état ! ’. Elle semblait me reprocher doucement l'excès de ma douleur ».

Muni d'un tel viatique, le petit orphelin se jette à la conquête de l'école, en classe de certificat d'études, puis en cours complémentaire. Pierre Besson livre à ce propos des pages d'anthologie, malheureusement peu connues de ceux que continue à fasciner l'école de la Troisième République, ce tome français de quelque encyclopédie gratuitement délivrée aux fous du savoir jusqu'au fin fond des montagnes et des vallées du Cantal. Un hommage vibrant est rendu aux maîtres, pour leur désintéressement dans le travail. Voici celui de la classe du certificat : " ce vaillant qui faisait dix heures de classe par jour, tenait gratuitement école le jeudi, se privait de toute récréation et consacrait ses longues veilles à la correction de nos malheureux cahiers, pour trente-six sous par jour, juste de quoi ne pas mourir de faim ». Voici maintenant le jeune directeur du cours complémentaire :
"Debout bien avant le jour, pour corriger nos devoirs et préparer ses leçons, il devait encore, avant de rentrer en classe, faire lever ses trente caméristes, les surveiller à l'étude du matin, les faire déjeuner. En classe, c'était une autre affaire: interroger, expliquer, répéter, réprimander, tancer, criailler, toute la journée. Le soir, étude, surveillance du souper, de la récréation, du dortoir. Le jeudi matin, classe ; le jeudi soir, promenade; le dimanche matin, surveillance, quasi obligatoire à cette époque, des exercices religieux; le dimanche soir, promenade. Enfin, le secrétariat de la mairie, prolongeant ses veilles et absorbant ses rares loisirs, lui donnait le coup de grâce. Il avait plus d'une fois les yeux rouges et cerclés de bistre".

Quant à l'enseignement délivré à ces futurs instituteurs de treize ou quatorze ans, il a les outrances, mais aussi la grandeur, des temps qui croyaient à l'encyclopédisme et au parcœur. L'épopée napoléonienne, les accidents du sol lorrain (la France a perdu la guerre de 
1870 par la géographie, on le sait bien, alors), les affluents de la Vilaine, les fleuves côtiers de la Manche ou de la Méditerranée, n'ont pas de secrets pour les candidats au certificat. "Le plus ignorant aurait pu citer sur les côtes une bonne centaine de golfes, de baies, de havres, d'anses, de caps, de promontoires, de pointes, de détroits, de goulots, de pertuis, d'îles et d'îlots ». Oui, comme le remarque l'auteur, "c'était assurément quelque chose d'original que l'existence, il y a vingt ans, dans un coin perdu de l'Auvergne, à quarante kilomètres de toute voie ferrée, d'une école où l'on étudiait avec une telle fureur ». La fureur de lire, déjà. Et celle d'écrire sans fautes, au sommet de ce rituel national qu'était la dictée :

«Et quelles dictées! Des dictées semées de mots traîtres, remplies de traquenards où nous butions à chaque pas. Pendant trois quarts d'heure, nous restions là, penchés sur notre cahier, nous martelant la cervelle, perdant les yeux à chercher les fautes, tournant et retournant les phrases, les propositions, les traduisant en patois, conjuguant les verbes, épelant les noms, scrutant les genres, surveillant les participes, suspectant les mots d'usage, pris de la fièvre du doute pour le plus commun des vocables - village, faut-il deux [l] ou un [1] ? - écrivant alors le mot suspect de trois ou quatre façons sur la couverture ou la table et choisissant celle qui choquait le moins l'œil; balbutiant tout bas des règles apprises par cœur - apaiser, apitoyer, aplanir, apercevoir, apostropher, apostiller ne prennent qu'un [p] - puis, lorsque le maître relisait, épiant les liaisons révélatrices qui lui échappaient à son insu, relisant nous-mêmes après lui, une fois, deux fois, une fois pour les fautes, une fois pour la ponctuation, utilisant jusqu'à la dernière minute, suppliant le maître de nous accorder une seconde, rien qu'une ».

\section{Des balcons en montagne, ou l'aventure du premier poste}

Heureux pays, réel et tout imaginaire à la fois ${ }^{25}$, que celui qui sut enchanter ses enfants dans un tel univers de devoir ludique et de jeux savants! Quels foyers d'images et de couleurs ont dû incarner, des décennies durant, avec leurs cartes, leurs atlas, leurs livres, leurs mots, ces écoles multipliées par la République jusque dans les hameaux, avec, si j'en crois l'exemple de Cévennes, leurs façades crépies de blanc aux embrasures régulières, luisant dans la pénombre schisteuse des villages comme le font, aujourd'hui, si banalement, les écrans de télévision dans les salons modernes! Seules les églises d'avant Vatican II (on en revient toujours au parallèle) offraient, de leur côté, une pareille accumulation de rouges et d'ors : avec la profusion des statues sulpiciennes, l'apparat des cérémonies (dont les fêtes annuelles de la Sainte-Enfance, marquées par de petites représentations théâtrales), ou la collection illustrée, à vocation mondiale, des Annales de la Propagation de la Foi. Le kitsch se déclinait au religieux (Thérèse de Lisieux dans ses pastels) comme au républicain (Marianne et les calligraphies?). Oui, les enfants rêveurs n'avaient que l'embarras du choix entre les deux imaginaires, également servis par des champions désintéressés et avides de se reconnaître dans des recrues arrachées à l'indigence, à l'ignorance et au mauvais temps des bergers.

Reste, pour l'écolier modèle devenu normalien, à prendre connaissance de sa première affectation, et à se rendre dans son école-appartement. Il s'agit toujours d'un poste situé aux limites: en altitude, dans les neiges, loin des villes et des grands axes, souvent à la frontière du département - et du temps. Juliette Bastide, Marcel Boyer, Adrienne DurandTullou, après Antonin Lavergne, nous ont laissé ce récit de l'arrivée dans le premier poste. Cela sonne comme autant de chapitres scolaires d'un Balcon en forêt que Julien Gracq aurait installé aux confins des Causses ou de la Margeride. Voici d'abord Michel Bastide (aliasMarcel Boyer) apprenant sa nomination, depuis sa bonne ville de Marvejols : 
"Enfin arriva l'enveloppe tant attendue portant l'en-tête de l'Inspection académique de la Lozère. Fébrilement, Maman ouvrit et vint me distraire pendant quelques instants de mon travail. Elle avait l'air inquiet. Je pris la feuille blanche et lus : \Monsieur Michel Bastide est nommé, à compter du $1^{\text {er }}$ octobre 1926, instituteur stagiaire à La Bessière, commune du Mazel (Lozère) \. ‘ La Bessière? Le Mazel ? Mais où est-ce donc? ’, demandait ma mère. Je n'en savais pas plus qu'elle![...] « Michel est nommé instituteur à La Bessière. Savez-vous où cela se trouve ? - La Bessière ? La Bessière ? Je connais un village qui s'appelle ainsi dans la commune de Javols `, avait dit l'un. « Il y en a un autre près de Ribennes ou Lachamp >, avait annoncé un second. Mais personne ne savait exactement où se trouvait Le Mazel. Les cartes de la région que je consultais montraient pourtant un chef-lieu de commune portant ce nom, au nord du département. [...] J'étais atterré. La perspective d'une installation en milieu rural ne m'effrayait pas, mais je n'avais rien prévu pour vivre plusieurs jours ou plusieurs semaines seul dans un village isolé».

Voici maintenant Adrienne Durand-Tullou à Nîmes, en décembre 1937, alors qu'elle a déjà passé huit mois près de La Bastide, en Lozère :

"Je rêvassais ce dernier matin de décembre 1937 quand la porte s'entrebâilla et une enveloppe jaune apparut, tendue à bout de bras: ‘ Tiens, c'est pour toi 〉, et la porte se referma. Instantanément, je me levai. L'enveloppe vola en morceaux. Un imprimé administratif avec des blancs remplis à la main : « 3 janvier 1938 > et « Rogues 〉. Ça y était ! J'avais ce que je voulais. La première exaltation tombée, je relus attentivement. Rogues? Connais pas. Je me précipitai sur le calendrier des P.T.T. Suivant les colonnes d'un index fébrile, je m'arrêtai à une ligne ‘ commune, 115 habitants, agence postale, téléphone `. [...] Je n'étais guère avancée. Une seule certitude: l'éloignement de Nîmes! Je pris la carte bien décidée à en tirer le maximum d'informations. Je remarquai d'abord Le Vigan pourvu $d u$ gros rond noir des sous-préfectures, puis Alzon, un chef-lieu de canton. Quant à Rogues, il figurait sur un fond jaune comme celui utilisé pour la garrigue. Aucune cote d'altitude. Une voix me soufflait : ‘ Tu voulais partir à la campagne pour y exercer ton métier, tu pars ; 115 habitants, ce n'est pas une capitale ; dans quatre jours tu auras des élèves, alors, tu verras bien > ».

Commence bientôt le voyage, ferroviaire, car la France des écoles rurales et des calendriers des P.T.T. permettait encore de se rendre en train au Vigan : « Au cours de deux brefs arrêts, j'eus la sensation d'avoir abandonné un univers accueillant et facile et de pénétrer dans un autre, sévère et énigmatique. La pluie fine s'était transformée en averses glaciales. Le train se vida rapidement de ses quelques voyageurs".

Le reste, c'est au lecteur de le découvrir, en rejoignant la cohorte de ceux qui ont fait du Pays des asphodèles un très grand, et très mérité, succès. Le reste, autobiographique, ne comprend guère plus que les travaux et les jours d'une institutrice rurale. Guère plus : le récit d'une incardination totale et définitive aux Causses gardois, si je peux emprunter ce mot au vocabulaire des carrières cléricales. Et les débuts puis l'épanouissement d'une vocation d'ethnologue et d'historienne qui a permis à la petite institutrice, mariée sur place, de devenir une grande dame et de composer une œuvre originale. Le pays des asphodèles montre, une fois de plus, que la tentation romanesque n'est jamais très éloignée de ces autobiographies d'instituteurs. Non pas le roman populaire (de qualité), façon "École de Brive ", qu'incarne désormais, pour l'école et les Cévennes L'année du certif, de Michel Jeury (1995), rapidement adapté à la télévision. Mais un roman de l'espace et des confins (géopolitique, ose Yves Lacoste à propos du Rivage des Syrtes), une réflexion sur la dimension spirituelle de l'attente et du (D)désert: on songe à Gracq, Buzzati, Giono, à Jean Carrière aussi. Le personnage principal du Rivage des Syrtes aime à rêver dans la salle des cartes : ainsi Marcel Boyer ou Adrienne Durand-Tullou devant le calendrier des P.T.T. 
ne saurait, peut-être, assez se féliciter des décisions prises par l'administration de l'Éducation nationale dans les années trente et cinquante: en faisant de tout jeunes normaliens des confinati abandonnés aux marches du Massif Central, elle a révélé des êtres à eux-mêmes, et fait s'épanouir des vocations dans la vocation. L'histoire de l'art a retenu le douanier Rousseau, le facteur Cheval : gageons qu'une enquête attentive aurait tôt fait de révéler de véritables créateurs ou penseurs chez les instituteurs ruraux - y compris dans les années 1970, qui drainèrent, à plusieurs reprises autour des dernières écoles de hameau, une si féconde agitation dans les hautes terres menacées par le silence.

\section{NOTES}

1. BERTOL, "L'instituteur doit être soldat ", in L'Auvergnat de Paris., 12 septembre et 3 octobre 1886. De LAS CASES E., Les élections et la paix sociale, extrait de La Réforme sociale, $1^{\text {er }}$ février 1904 , p.14.

2. Un modeste monument a été élevé aux deux jeunes filles au lieu-dit La Vaissière, sur la route allant du Pont-de-Montvert au col de Montmirat sur la RN 106. Le collège Marthe-Dupeyron, à Langogne, perpétue le souvenir de cette fille d'instituteurs, née en 1919 à Rodes, en poste à l'école de La Vaissière, à 1200 mètres d'altitude, depuis le mois d'octobre 1940. L'émotion fut immense en Lozère, et ne s'est pas éteinte. Cf., BUFFIÈRE P., « Dupeyron (Marthe et Pierrette) », in BUFFIÈRE F., dir., Lozériens connus ou à connaître, Toulouse, 1992, p. 260-262.

3. Éd.: Mercure de France, 198 p. Le livre est dédié à Simone de Beauvoir, et rappelle l'intervention de l'Académicien André Maurois, cousin par alliance de François de Fiers (conseiller général du canton du Malzieu), pour obtenir de la municipalité du Malzieu-Forain qu'elle fasse mettre l'eau à l'école du hameau des Ducs.

4. Les Éditions de Paris, 1995, 326 p. J'ai eu l'honneur de présenter cet ouvrage (Cf., la « Préface », p. 7-12).

5. MARCHAL G.-L., Les Hussards Verts. La condition d'instituteur dans les monts de Lacaune à mi-XX siècle. Castres, Société Culturelle du Pays Castrais, 1994, 270 p.

6. BRAJON M., Monsieur Brajon, maître d'école, Paris, 1977. L'auteur a reçu sa formation dans les établissements des Frères des Écoles Chrétiennes, mais a renoncé à entrer dans la congrégation, tout en enseignant dans des écoles catholiques. Toinou, le cri d'un enfant auvergnat, d'Antoine SYLVÈRE (Paris, 1980) est aussi un témoignage de première main sur les écoles dirigées par les Frères des Écoles Chrétiennes.

7. Voir mon livre, qui comporte un chapitre sur les instituteurs : Cadets de Dieu. Vocations et migrations religieuses en Gévaudan, XVIII ${ }^{e}$-XX ${ }^{e}$ siècle, Paris, CNRS Éditions, 1997.

8. Des témoignages avérés rapportent toutefois que l'ordre de mérite au catéchisme est souvent truqué, afin de donner les premières places aux enfants des notables - et bienfaiteurs de la paroisse.

9. Archives Départementales de la Lozère (ADL), 1 T 840, «Curés enseignants aux jeunes gens dans les paroisses ".

10. D'après une enquête orale auprès de la prieure du carmel de Mende, 1988.

11. "On le voyait, pendant ses tournées de confirmation, chercher partout les jeunes samuel qui pouvaient être appelés de Dieu; à l'église, à l'école, dans les maisons, sur les chemins, quand un enfant frappait son regard observateur par son intelligence et sa candeur, il le fixait un instant, le marquait au 
front, et avec sa voix solennelle qu'il voulait rendre prophétique : ‘ Toi, disait-il, tu seras vicaire ici, toi, tu seras curé là >. Et il semait un peu partout de ces futurs curés ou vicaires que son zèle cherchait à multiplier dans toutes les paroisses ", in : RICARD E., Le cardinal Bourret. Souvenirs intimes, Paris, 1897, p. 186. L'Aveyronnais Gély, évêque de Mende à partir de 1906, imita systématiquement le cardinal Bourret.

12. BUFFIÈRE F., dir., Lozériens connus ou à connaître, op. cit.. p. 237-238. La Lozère nouvelle, 25 décembre 1987.

13. « « Tenez, vous devriez le mettre à Barsac, au Cours Complémentaire. Vous verrez, il sera reçu au certificat, votre Henri. Je parlerai au Directeur. Il en a trois des miens déjà , explique l'institutrice à la mère", in Henri Gouttebel, instituteur, Clermont Ferrand, 1971, vol. 1, p. 40. MAZAURIC L., Belle rose, ô Tour Magne, Paris, 1969. (Sur son père, né à Valleraugue dans les Cévennes gardoises).

CARLES E., Une soupe aux herbes sauvages, Paris, 1977, p. 61-62, (Alpes). CORNEC J., Josette et Jean Cornec instituteurs. De la hutte à la lutte. 1886-1980, Paris, 1981. (Sur la mère de l'auteur, Josette). Marthe BOISSIER, née en 1877 à la Salle-Prunet (Lozère), devenue directrice d'École normale et poétesse, doit aussi son destin à l'insistance d'un instituteur.

14. Jean Coste, Cahiers de la Quinzaine, 1903, p. 166-167. On trouve la réimpression à l'identique du roman dans l'édition critique par Anne ROCHE de Charles PÉGUY, De Jean Coste, Paris, Éd. Klincksieck, 1975.

15. Cité par le Bulletin de l'Instruction primaire du département de la Lozère, avril 1892.

16. ADL, 1 T 8, 1900, p. 495 (rapport de l'inspecteur) et 1 T 726 (dossier personnel de BASTIDE J.).

17. Chiffres établis à partir du dossier $1 \mathrm{~T} 763$ des ADL.

18. Ozouf M., "Présentation: l'image dans le tapis», in L'école de la France. Essais sur la Révolution, l'utopie et l'enseignement, Paris, Gallimard, 1984. p. 7-24.

AGULHON M., " Vu des coulisses », Pierre Nora, présente, Essais d'ego-histoire, Paris, Gallimard, 1987, p. 10-11.

19. Utilisés dans deux ouvrages, Nous les maîtres d'école, Paris, Éd. Julliard, 1967, et La République des Instituteurs. Paris, Gallimard/Le Seuil, 1992.

20. Le père de Jean-François Roux refuse de le laisser partir à Paris, et lui prédit qu'il y serait « plus malheureux que les pierres", et "qu'il se repentirait tôt ou tard de n'avoir point écouté les conseils paternels ", ce qui fut le cas, dans un premier temps.

21. Jean-François est le cinquième de neuf enfants ; cadet, il peut gagner Paris, et y entraîner un jeune frère. Mais leur aîné, Claude, a appris le métier de scieur de long avec le père, et habite la maison familiale, "pour perpétuer le berceau de la famille, et en transmettre le culte à ses enfants ", se réjouit l'auteur, qui n'a pourtant pas voulu de ces filiations.

22. Ne soyons pas dupes: il entre dans ces textes une indéniable tension littéraire, à l'aune de l'aventure exceptionnelle vécue par leur auteur. Mais il n'était pas convenable, alors, pour un " primaire ", d'oser la forme romanesque, et c'est sans doute ce qu'Antonin Lavergne a dû expier en partie : alors que l'on pouvait avancer sans risques, à couvert de l'autobiographe moralisante. 23. $1903 / 1$, p. $476-487$, et $1904 / 2$, p. 153-173. Un caméristat était un internat sommaire dans lequel les élèves fournissaient les draps et leur nourriture, voire le bois de chauffage; remarquablement adaptés aux conditions socio-économiques et de circulation dans le Massif Central, les caméristats y ont été très répandus, notamment dans les écoles catholiques. Le Dictionnaire de Pédagogie de Ferdinand BUISSON les a jugés suffisamment importants pour leur consacrer un article (I, 1, p. 322).

24. Revenons un instant sur le parallèle avec l'Église : l'univers missionnaire, à son apogée entre 1860 et 1940, a su séduire, sur les mêmes terres, d'autres enfants grandis dans la misère et les «loues». Citons deux grands témoignages, trop peu connus, écrits l'un par un Oblat de Marie (que sa santé empêcha de partir dans le Grand nord canadien, comme il s'y était préparé), l'autre par un Spiritain (que sa congrégation utilisa dans ses maisons de formation, plutôt que de 
l'envoyer en Afrique noire ou en Amazonie) : ROCHE A., Granit et amour. Une enfance lozérienne, Paris, Sherbrooke, 1972 ; BOISSET F., Voyage et fantaisie. Mémoires d'un naïf, Allex, 2 vol., 1973 et 1980.

25. Voir la très belle réflexion du sociologue Pierre SANSOT, à la gloire notamment de la dictée : «La France, un fait d'imagination?", in Cahiers internationaux de Sociologie, Vol. LXXXIV. 1988, p. 135-149.

\section{RÉSUMÉS}

Non disponible

Not available

INDEX

Mots-clés : école primaire, IIIe république, instituteur, Languedoc, ruralité

Keywords : primary school, rural context, rural life, school teacher, third republic

\section{AUTEUR}

\section{PATRICK CABANEL}

Université de Toulouse Le Mirail 